\title{
Comparison of thigh muscle activations in single leg exercises: bench squat, step-up, airborne lunge
}

\author{
Fahri Safa Çinarli ${ }^{1 \mathrm{ABCDE}}$, Sena Çinarli ${ }^{2 \mathrm{ABD}}$, Emin Kafkas ${ }^{1 \mathrm{ABD}}$ \\ ${ }^{1}$ Inonu University, Faculty of Sport Sciences, Department of Movement and Training Science \\ ${ }^{2}$ Inonu University, Faculty of Physical Therapy and Rehabilitation
}

Authors' Contribution: A - Study design; B - Data collection; C - Statistical analysis; D - Manuscript Preparation; E - Funds Collection

\begin{tabular}{|c|c|}
\hline \multicolumn{2}{|l|}{ Abstract } \\
\hline $\begin{array}{l}\text { Background } \\
\text { and Study Aim }\end{array}$ & $\begin{array}{l}\text { Single leg exercises have some advantages in terms of time, practice and energy costs. However, the } \\
\text { activation values that occur in different single leg exercises can be used for training planning. The aim of } \\
\text { this research was to examine the thigh muscle activation values during three different single leg exercises. }\end{array}$ \\
\hline $\begin{array}{l}\text { Material and } \\
\text { Methods }\end{array}$ & $\begin{array}{l}\text { Ten healthy male volunteers who were students of the faculty of sports sciences participated in the study. } \\
\text { In the study, the EMG ampilitude values of the vastus medialis (VM), vastus lateralis (VL), semitendinosus } \\
\text { (SEM) and biceps femoris (BF) muscles were examined during Step-up, Bench Squat and Airborne Lunge } \\
\text { exercises. At the same time, Quadriceps (VM+VL): Hamstring (SEM+BF) ratios were determined. }\end{array}$ \\
\hline Results & $\begin{array}{l}\text { Significant differences were detected in all thigh muscles in the ascent and descent phases }(p<0.05) \text {. While } \\
\text { the greatest activation for the quadriceps group was seen in the airborne lunge, the greatest activation } \\
\text { for the hamstring group was detected in the bench squat. A statistically significant difference was found } \\
\text { in terms of exercise practices in the quadriceps: hamstring }(\mathrm{Q}: \mathrm{H}) \text { ratio }(\mathrm{F}(2,18)=12.282, \mathrm{p}=.003) \text {. It was seen } \\
\text { that the most balanced exercise was bench squat }(\mathrm{Q}: \mathrm{H}=2.55) \text {, and the most unbalanced exercise (agonist } \\
\text { dominant) was airborne lunge ( } \mathrm{Q}: \mathrm{H}=5.51) \text {. }\end{array}$ \\
\hline Conclusions: & $\begin{array}{l}\text { The findings show that the exercises examined can be selected depending on the purpose of the training. } \\
\text { While bench squats can be preferred for more balanced co-activation the airborne lunge can be preferred } \\
\text { for dominant knee extensors. }\end{array}$ \\
\hline words: & \\
\hline
\end{tabular}

\section{Introduction}

The effects of single leg exercises on both performance and health have been examined. In a study, it was mentioned that single leg exercises have positive effects on regional arterial stiffness [1]. In another study, it was determined that these exercises abolished the impaired oxidative flow in the skeletal muscle of patients with type 2 diabetes and, at the same time, increased the vascular endothelial content [2]. It has been mentioned that single leg exercises are also applied by clinicians during the rehabilitation process and that they have an effect on improving aerobic capacity by reducing metabolic demand [3].

Single leg exercises are frequently examined in biomechanical studies in order to make more isolated analyzes in terms of the skeletal-muscular system [4]. In addition, it has been stated that single leg exercises are superior to double leg exercises in terms of energy metabolism [5]. High-intensity exercises are limited by hematological or cardiovascular factors rather than the oxygen capacity of the working muscle, due to the high oxygen demand or the heavy load it creates on the organism $[5,6]$. At this point, it is noted that the amount of intense oxygenated blood required by the working muscles is more tolerable during exercises involving one leg or smaller muscle mass [7]. For this reason, it can be said that single leg exercises have the advantages of energy cost and sustainable exercise.

It is necessary to compare different practices in order to design the right exercises for strength and conditioning training. The appropriate exercise choice is possible using the activation values determined during different exercises for the same muscle or muscle group $[8,9]$. The findings obtained may be followed by exercise participants for their current training situations or targeted goals. At the same time, especially for clinicians, co-activation values serve as an important feedback function in the rehabilitation process and as an injury prevention strategy [10, 11]. For these and similar purposes, comparison of exercises can help in choosing the right exercise.

It is known that sagittal plane exercises are given priority in the exercise programming process [12]. It can be said that the axis in which the walking pattern is exhibited and for which the least foreignness can be felt by the organism is stepping. For this reason, stepping movements in the sagittal plane are practiced by many exercise participants. In this context, the aim of the research was to examine the thigh muscle activations during different single leg exercises in the sagittal plane. In the research, bench squat, step-up and airborne lunge movements with different difficulty levels but mainly including ascending and descending phases were examined. The hypothesis determined that the thigh muscle activity values differ in terms of exercises. 


\section{Material and Methods}

\section{Participants}

Ten healthy male volunteers (age: $26.7 \pm 3.65$ years; body weight: $77 \pm 11.4 \mathrm{~kg}$; height: $168.2 \pm 27.86 \mathrm{~cm}$ ) who were students of the faculty of sports sciences participated in the study. The study was approved by the Ethics Committee of Inonu University (Approval Number: 20212166), and conformed to the Helsinki Declaration. Written informed consent was obtained from all participants. Inclusion criteria in the study were determined as not having any musculoskeletal disorders, having at least 3 years of bodybuilding and fitness experience, and having experienced the movements before the research. Exclusion criteria were determined as experiencing any health problems, kinematic errors during the display of movement, and incorrect data measurement in activation values.

\section{Research Design}

Exercise Procedure

In the research, bench squat, step-up and airborne lunge exercises were examined and the movements were analyzed as ascending and descending phases. The participants were randomized to the exercises using a computer-generated randomization list. The movements were repeated three times, and the most correct posture was analyzed after the video review (Figure 1). Although different movement patterns were examined, a goniometer was used to standardize the flexion and extension angles of the knee. The knee flexion angle was maintained at 90 degrees at the starting point of the movement, and the knee was in full extension at the end of the ascending phase of the movement. Exercises were applied at the pace that the participants chose, so that the flow of the exercises was not disturbed.

\section{Electromyography (EMG) Procedure}

Electrode placements followed SENIAM recommendations and were applied to the dominant leg [13]. Before the electrodes were positioned over each muscle, the skin was prepared by shaving, abrading, and cleaning with isopropyl alcohol wipes to reduce skin impedance values. Following the skin preparations, circular bipolar Ag-AgCI surface electrodes (Noraxon Dual Electrodes, Noraxon USA, Scottsdale, Arizona, USA) (diameter $=1 \mathrm{~cm}$ and interelectrode distance $=2 \mathrm{~cm}$ ) were placed on the volunteer's right side [14]. Vastus medialis (VM), vastus lateralis (VL), biceps femoris (BF) and semitendinosus (SEM) muscles of participants were examined. In addition, Quadriceps (VM+VL):Hamstring $(\mathrm{BF}+\mathrm{SEM})$ ratio $(\mathrm{Q}: \mathrm{H})$ was determined.

EMG Signal Processing

Raw sEMG signals were collected using an 8-channel wireless telemetry system (Noraxon Desktop DTS, Scottsdale, AZ, USA) and were analyzed using MyoMuscle MR 3.10 Clinical Applications software (Noraxon Telemyo, Scottsdale, AZ, USA). All raw sEMG signals were first 20-500 Hz Butterworth bandpass filtered, and then root-mean square (RMS) filtered with a $100 \mathrm{~ms}$ time-window for movement artifact rejection and signal smoothing. RMS-filtered mean EMG signal of exercises was expressed as microvolt $(\mu \mathrm{V})$.

Statistical Analysis

Findings were analyzed using GraphPad Prism 7.0 software (GraphPad Software Inc, San Diego, California, USA). The statistical analysis was initially performed by the Shapiro Wilks normality test. Repeated measures ANOVA was used to determine exercise differences. If there was a difference between exercises, Bonferroni multiple comparison test was performed to find favorite exercise type. Significance level in the study was determined as $\mathrm{p}<0.05$. Results were presented graphically, including mean and standard deviations.

\section{Results}

The findings obtained in the research were shown as ascent phase, descent phase and $\mathrm{Q}: \mathrm{H}$ ratio. A significant difference was found in all thigh muscles in the ascent and descent phases (Table 1 and Table 2, respectively).

Figure 2 shows that the exercises differ significantly in terms of muscles in the ascending phase $(p<0.05)$. In the ascending phase, the highest activation for the VM and VL muscles was observed in the airborne lunge, while the highest activation for the SEM and BF was detected in the bench squat movement (Figure 2).

Figure 3 shows that the exercises differ significantly in terms of muscles in the descending phase $(p<0.05)$. In the descending phase, the highest activation for the VM and VL muscles was observed in the airborne lunge, while the highest activation for the SEM and BF was detected in the bench squat movement (Figure 3).

When the activation values throughout the exercise were examined (Table 3), it was found that the Q:H ratio differed statistically significant $(\mathrm{F}(2,18)=12.282, \mathrm{p}=.003)$. Bench squat, step-up and airborne lunge were found to be the most balanced exercises for the Q:H ratio, respectively (Figure 4). There was a statistically significant difference between bench squat and step-up and between bench squat and airborne lunge in terms of co-activation $(\mathrm{p}<0.01)$.

\section{Discussion}

In the study, thigh muscle activation values that occur during three different exercises were examined. Significant differences were detected in terms of thigh muscles in the ascending and descending phases of the exercises $(p<0.05)$. For the quadriceps group, the greatest activation was seen in the airborne lunge, while the greatest activation for the hamstring group was seen in the bench squat exercise. The most balanced exercise with the $\mathrm{Q}: \mathrm{H}$ ratio was the bench squat $(\mathrm{Q}: \mathrm{H}=2.55)$, while the most agonist dominant exercise was the airborne lunge $(\mathrm{Q}: \mathrm{H}=5.51)$.

In clinical studies, it has been mentioned that unilateral exercises have some advantages over bilateral exercises. Especially in acute studies, time and cost are shown in terms of increasing the statistical reliability of the research [15]. Unilateral exercises are preferred in order to optimize the energy cost and minimize the catabolic effect of the exercise [5]. It can be said that single leg or one leg exercises are advantageous in terms of metabolic 


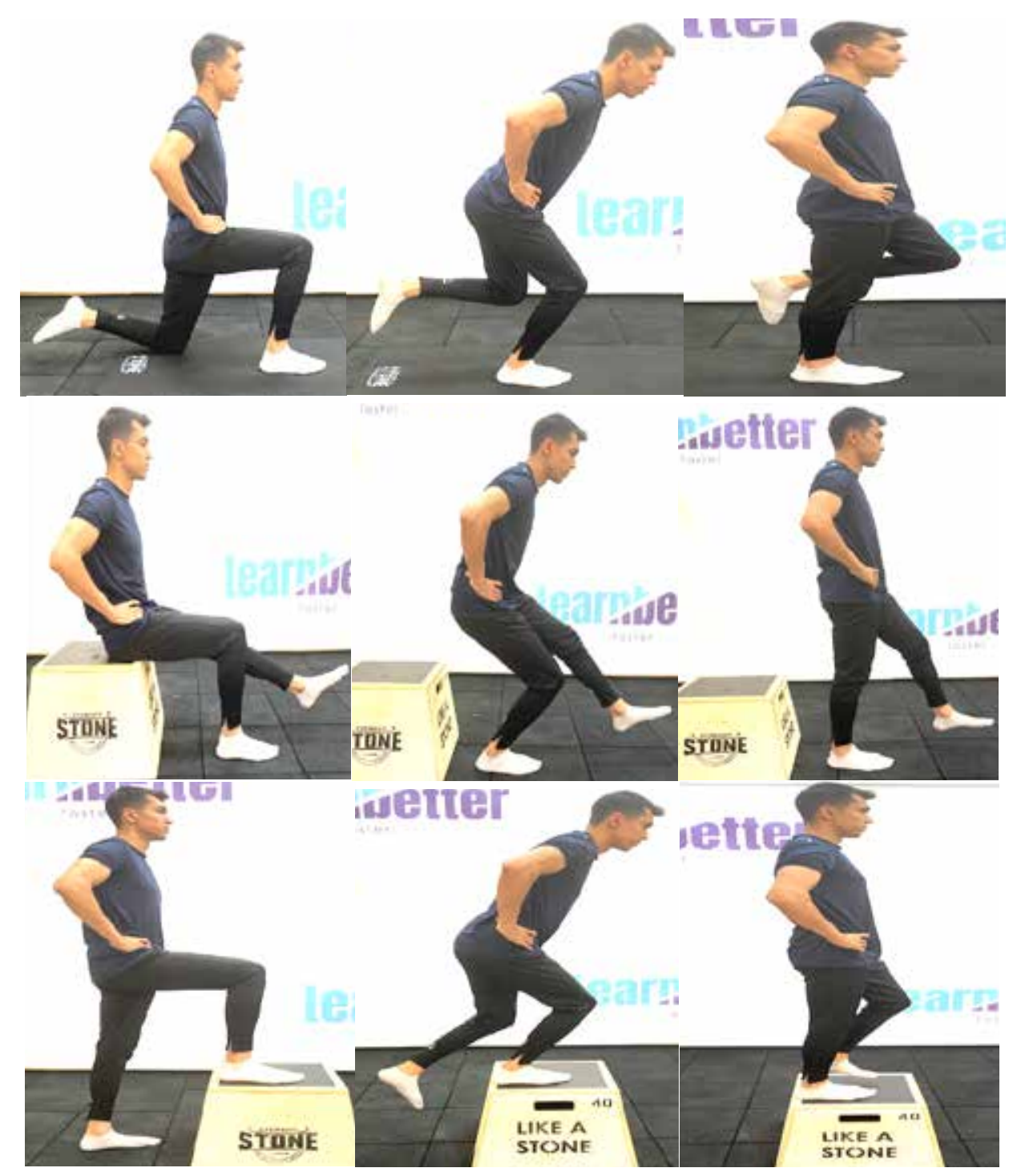

Figure 1. The beginning, middle and ending phases of exercises A) Airborne Lunge; B) Bench Squat; C) Step-up

Table 1. The mean RMS $(\mu \mathrm{V})$ values in the ascending phase during exercises

\begin{tabular}{llllll}
\hline Muscles & Exercises & Mean & SD & $\mathbf{F}_{(2,18)}$ & $\mathbf{P}$ \\
\hline \multirow{4}{*}{ VM } & Bench squat & 157.3 & 32.13 & & \\
& Step-up & 152.8 & 38.38 & 14.617 & $<0.01^{* *}$ \\
& Airborne lunge & 208.4 & 48.85 & & \\
VL & Bench squat & 169.7 & 29.51 & & $.028^{*}$ \\
& Step-up & 170.6 & 47.48 & 4.378 & \\
& Airborne lunge & 218.1 & 72.57 & & \\
SEM & Bench squat & 80.3 & 33.26 & & $<0.01^{* *}$ \\
& Step-up & 37.6 & 21.42 & 23.182 & \\
& Airborne lunge & 46.4 & 13.24 & & $<0.01^{* *}$ \\
& Bench squat & 53.9 & 30.99 & & \\
\hline
\end{tabular}

$\left({ }^{*} p<0.05 ;{ }^{*} p<0.01 ; \mathrm{VM}\right.$ : Vastus medialis; VL: Vastus lateralis; SEM: Semitendinosus; BF: Biceps femoris) 


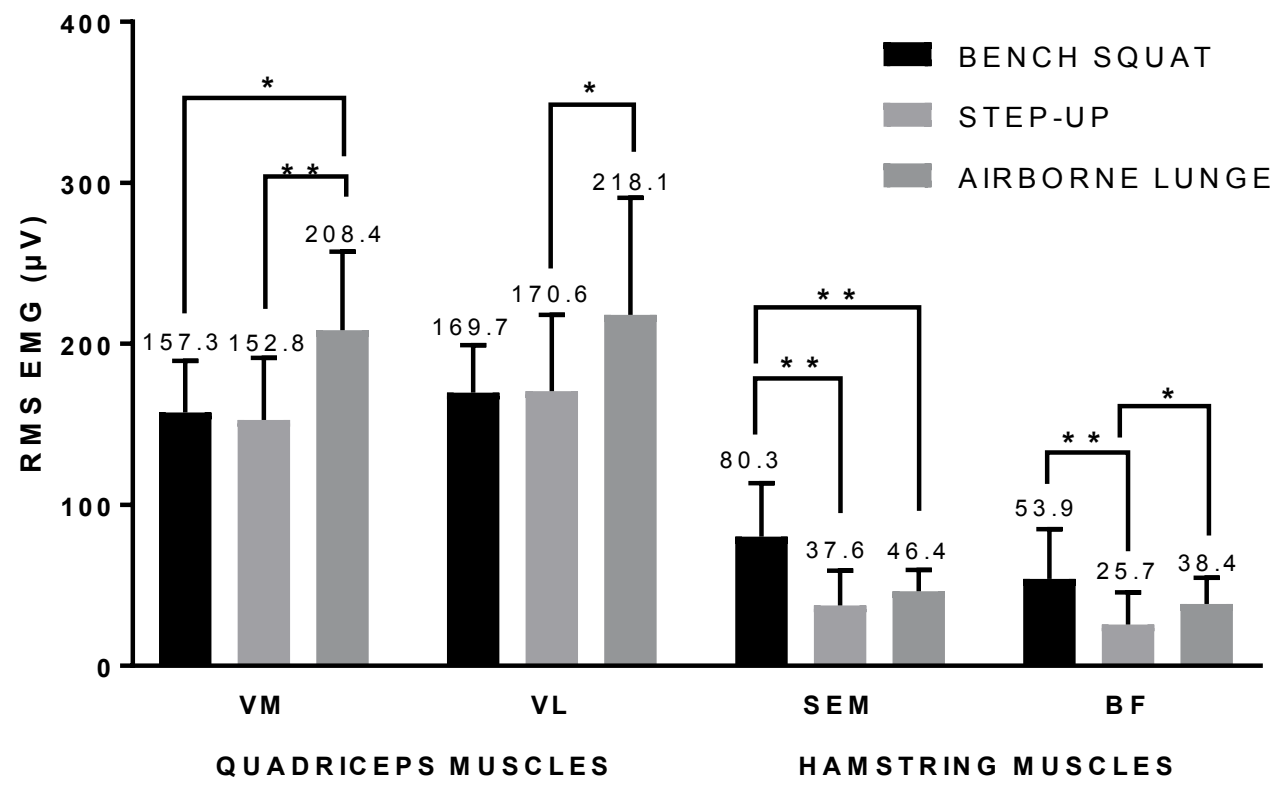

Figure 2. Multiple comparison of the RMS values in ascending phase $\left({ }^{*}: p<0.05 ;{ }^{* *}: p<0.01\right)$ : (VM: Vastus medialis; VL: Vastus lateralis; SEM: Semitendinosus; BF: Biceps femoris)

Table 2. The mean RMS $(\mu \mathrm{V})$ values in the descending phase during exercises

\begin{tabular}{|c|c|c|c|c|c|}
\hline Muscles & Exercises & Mean & SD & $\mathbf{F}_{(2,18)}$ & $\mathbf{P}$ \\
\hline & Bench squat & 122.2 & 29.76 & & \\
\hline \multirow[t]{3}{*}{ VM } & Step-up & 98.4 & 32.03 & 8.785 & $.013^{*}$ \\
\hline & Airborne lunge & 141 & 47.53 & & \\
\hline & Bench squat & 128 & 29.33 & & \\
\hline \multirow[t]{3}{*}{ VL } & Step-up & 106.7 & 34.36 & 10.700 & $.001^{* *}$ \\
\hline & Airborne lunge & 147.4 & 46.58 & & \\
\hline & Bench squat & 92.2 & 49.92 & & \\
\hline \multirow[t]{3}{*}{ SEM } & Step-up & 51.9 & 53.81 & 5.812 & $.011^{*}$ \\
\hline & Airborne lunge & 32.3 & 15.16 & & \\
\hline & Bench squat & 47.1 & 26.84 & & \\
\hline \multirow[t]{2}{*}{$\mathrm{BF}$} & Step-up & 14.7 & 12.80 & 15.396 & $.002^{* *}$ \\
\hline & Airborne lunge & 24.2 & 11.50 & & \\
\hline
\end{tabular}

( ${ }^{*} \mathrm{p}<0.05 ;{ }^{*} \mathrm{p}<0.01 ; \mathrm{VM}$ : Vastus medialis; VL: Vastus lateralis; SEM: Semitendinous; BF: Biceps femoris)

Table 3. $\mathrm{Q}: \mathrm{H}$ ratio of mean $\mathrm{RMS}$ values for the entire movement

\begin{tabular}{lllll}
\hline Exercise & VM+VL/SEM+BF & SD & $\mathbf{F}_{(2,18)}$ & P \\
\hline Bench squat & 2.55 & .94 & & $.003^{* *}$ \\
Step-up & 4.84 & 1.84 & 12.282 & .02 \\
Airborne lunge & 5.51 & 2.31 & & \\
\hline
\end{tabular}

( ${ }^{* *} \mathrm{p}<0.01 ; \mathrm{VM}$ : Vastus medialis; VL: Vastus lateralis; SEM: Semitendinosus; BF: Biceps femoris)

efficiency, time and cost. However, the detection of crosschain power transfer or the inability to detect dominantnon-dominant limb differences can be expressed as limitations for unilateral exercises.

In the study, the quadriceps group showed greater EMG amplitude in the airborne lunge exercise and the hamstring group showed this in the bench squat exercise. The findings may be due to the different difficulty levels of the exercises, as it has been mentioned that there is a relationship between Surface EMG values and motor unit activation, and EMG amplitude depends on the number of motor unit activations, size and firing rate 


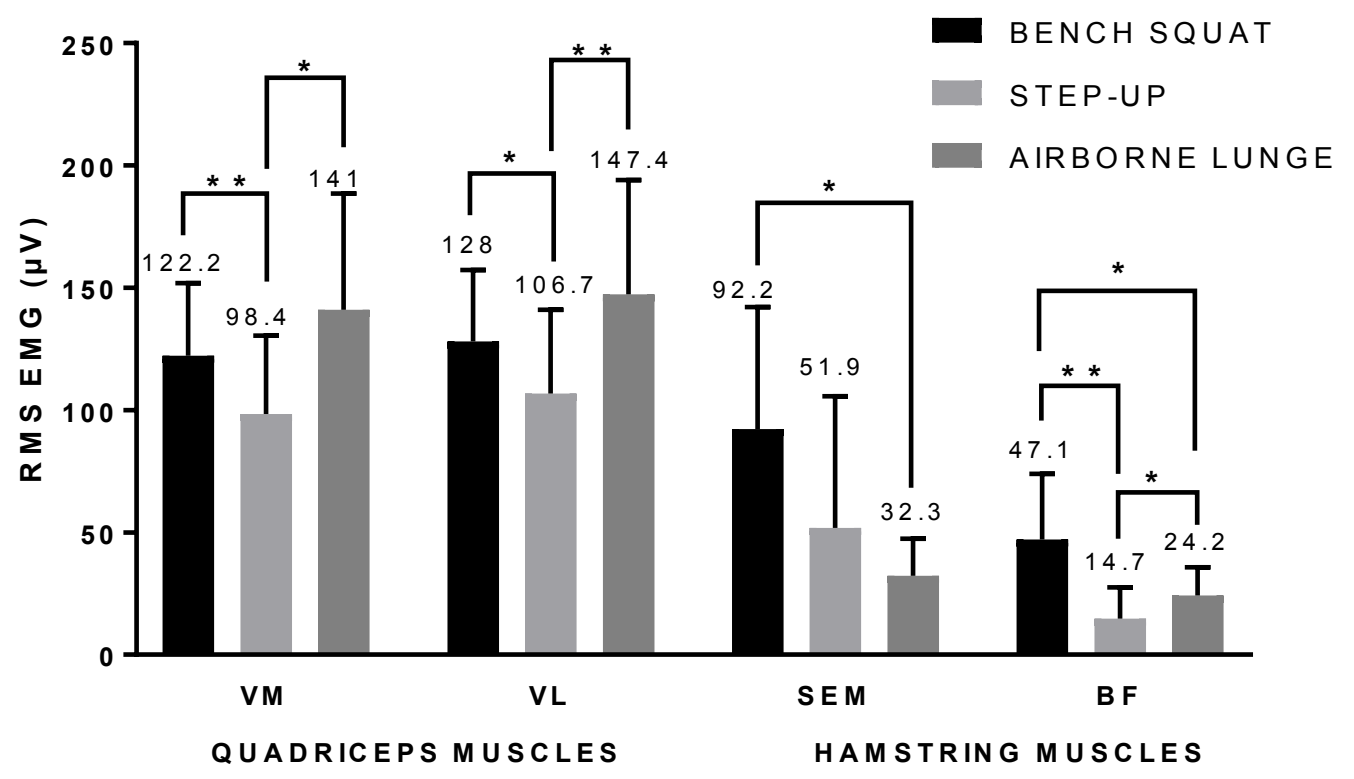

Figure 3. Multiple comparison of the RMS values in descending phase $\left({ }^{*}: p<0.05 ;{ }^{* *}: p<0.01\right)$ : (VM: Vastus medialis; VL: Vastus lateralis; SEM: Semitendinosus; BF: Biceps femoris)

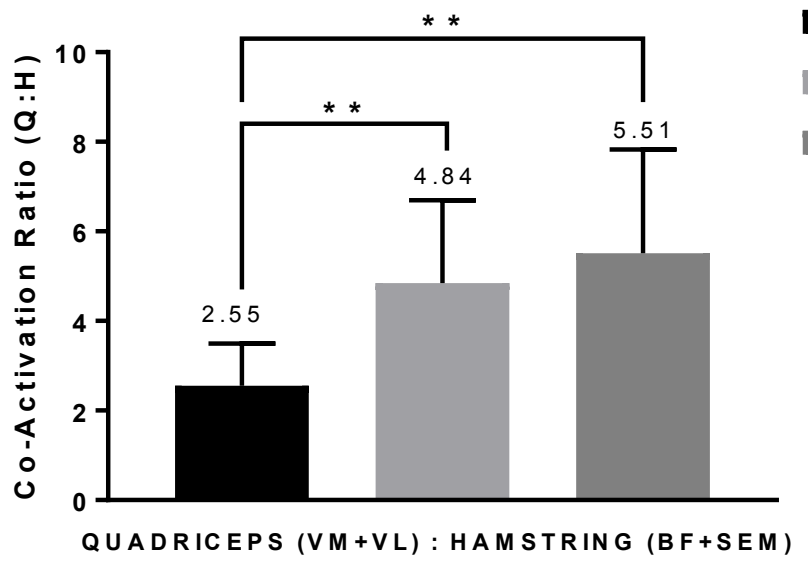

BENCH SQUAT

STEP-UP

AIRBORNE LUNGE

Figure 4. $\mathrm{Q}: \mathrm{H}$ ratio of the entire exercise $\left({ }^{* *} \mathrm{p}<0.01\right)$ : (VM: Vastus medialis; VL: Vastus lateralis; SEM: Semitendinosus; BF: Biceps femoris)

[16]. It has been stated that the increased value in the amplitude is an interpretable parameter in terms of the motor unit participation rate [17]. Furthermore, it has been determined that quadriceps and hamstring muscles produce higher EMG activity during high resistance in low and high resistance training practices [18]. As the need for muscle strength increases, compensation is provided to maintain the movement and increases in signal values can be seen. Therefore, the quantitative activation values seen during the comparison of different exercises can be an important parameter in determining the exercise goal. Biomechanically, the airborne lunge includes the movement between kneeling and standing. Since the body's center of gravity is closer to the ground during this exercise, the process of standing up can be more challenging for the agonist muscle, the movement between sitting and standing in the bench squat movement.
During this movement, the contact of the gluteus maximus with the bench in the starting position may have differentiated the standing up process compared to the other two exercises. When getting up from the sitting position, the hamstring muscle group in the antagonist role may have been more active for balancing. Depending on the level of difficulty, it can be stated that the exercises require different levels of motor unit participation.

In terms of the thigh muscle group, the $\mathrm{Q}: \mathrm{H}$ ratio may affect exercise preference [19]. Greater hamstring activation, especially in the clinical rehabilitation phase, is a protective response to limit anterior tibial translation of the knee after injury and increase joint stability [20, 21]. It has been stated that single leg exercises can reduce the risk of tensile stress on the ACL when performed at a 30-90 degree knee flexion angle [10]. In the research, it was determined that the most balanced Q:H ratio was in 
the bench squat movement and the most unbalanced ratio was in the airborne lunge movement.

\section{Conclusions}

The activation values obtained from the exercises examined can be selected according to the purpose of the exercise to be planned. While airborne lunge can be preferred especially in quadriceps dominant exercises, bench squat can be applied in terms of the hamstring group. Finally, when the Q:H ratio is important, it can be said that the bench squat movement can be safer, especially in terms of the balanced co-activation value demanded during the rehabilitation process.

\section{Conflict of interest}

The authors declare no conflict of interest.

\section{References}

1. Heffernan KS, Rossow L, Jae SY, Shokunbi HG, Gibson EM, Fernhall B. Effect of single-leg resistance exercise on regional arterial stiffness. European Journal of Applied Physiology, 2006; 98(2): 185-190. https://doi.org/10.1007/s00421-006-0259-9

2. Scalzo RL, Schauer IE, Rafferty D, Knaub LA, Kvaratskhelia N, Johnson TK, et al. Single-leg exercise training augments in vivo skeletal muscle oxidative flux and vascular content and function in adults with type 2 diabetes. J Physiol, 2021:JP280603. https://doi.org/10.1113/JP280603

3. Dolmage TE, Goldstein RS. Effects of one-legged exercise training of patients with COPD. Chest, 2008; 133(2):370-376. https://doi.org/10.1378/chest.07-1423

4. Zwerver J, Bredeweg SW, Hof AL. Biomechanical analysis of the single-leg decline squat. British Journal of Sports Medicine, 2007; 41(4): 264-268. https://doi.org/10.1136/bjsm.2006.032482

5. Abbiss CR, Karagounis LG, Laursen PB, Peiffer JJ, Martin DT, Hawley JA et al. Single-leg cycle training is superior to double-leg cycling in improving the oxidative potential and metabolic profile of trained skeletal muscle. Journal of Applied Physiology, 2011; 110(5): 1248-1255. https://doi.org/10.1152/japplphysiol.01247.2010

6. Davies CT, Sargeant AJ. Effects of training on the physiological responses to one-and two-leg work. Journal of Applied Physiology, 1975; 38(3): 377-375. https://doi.org/10.1152/jappl.1975.38.3.377

7. Wezenberg D, de Haan A, van der Woude LH, Houdijk H. Feasibility and validity of a graded one-legged cycle exercise test to determine peak aerobic capacity in older people with a lower-limb amputation. Physical Therapy, 2012; 92(2): 329-338. https://doi.org/10.2522/ptj.20110125

8. Earl JE. Gluteus medius activity during 3 variations of isometric single-leg stance. Journal of Sport Rehabilitation, 2005; 14(1): 1-11. https://doi.org/10.1123/jsr.14.1.1

9. Çınarlı FS, Ölmez SB, Namaldı S, Karanfil E, Güllü K, Soylu AR. The examination of thigh muscle activations in bridgeplankexercisesperformedondifferentgrounds.TurkishJournal of Physiotherapy and Rehabilitation, 2020; 31(2): 156-162. https://doi.org/10.21653/tjpr.547050

10.Dedinsky R, Baker L, Imbus S, Bowman M, Murray L. Exercises that facilitate optimal hamstring and quadriceps co-activation to help decrease acl injury risk in healthy females: a systematic review of the literature. International Journal of Sports Physical Therapy, 2017; 12(1): 3-15.

11.Selistre LFA, Mattiello SM, Nakagawa TH, Gonçalves GH, Petrella M, Jones RK. The relationship between external knee moments and muscle co-activation in subjects with medial knee osteoarthritis. Journal of Electromyography and Kinesiology, 2017; 33: 64-72. https://doi.org/10.1016/j.jelekin.2017.01.007

12.Fountaine CJ. Unilateral and bilateral exercise movements: Considerations for Program Design. ACSM's Health \& Fitness Journal, 2018; 22(3): 11-16. https://doi.org/10.1249/FIT.0000000000000390

13.Project SENIAM. Surface Electromyography for the noninvasive assessment of muscles. [Internet]. 2011. [updated 2021 April 16; cited 2021 Jun 02]. Available from: www. seniam.org

14.Rota S, Rogowski I, Champely S, Hautier C. Reliability of EMG normalisation methods for upper-limb muscles. Journal of Sports Sciences, 2013; 31(15): 1696-1704. https://doi.org/10.1080/02640414.2013.796063

15.MacInnisMJ,McGloryC,GibalaMJ,PhillipsSM.Investigating human skeletal muscle physiology with unilateral exercise models: when one limb is more powerful than two. Applied Physiology, Nutrition, and Metabolism, 2017; 42(6): 563-570. https://doi.org/10.1139/apnm-2016-0645

16.Suzuki H, Conwit RA, Stashuk D, Santarsiero L, Jeffrey Metter E. Relationships between surface-detected EMG signals and motor unit activation. Medicine \& Science in Sports \& Exercise, 2002;34:1509-17. https://doi. org/10.1097/00005768-200209000-00018

17.Fallentin N, Jørgensen K, Simonsen EB. Motor unit recruitment during prolonged isometric contractions. European Journal of Applied Physiology and Occupational Physiology, 1993; 67(4): 335-341. https://doi.org/10.1007/BF00357632

18. Schoenfeld BJ, Contreras B, Willardson JM, Fontana F, Tiryaki-Sonmez G. Muscle activation during low-versus high-load resistance training in well-trained men. European Journal of Applied Physiology, 2014; 114(12): 2491-2497. https://doi.org/10.1007/s00421-014-2976-9

19.Thomas AC, Judd DL, Davidson BS, Eckhoff DG, Stevens-Lapsley JE. Quadriceps/hamstrings co-activation increases early after total knee arthroplasty. The Knee, 2014; 21(6): 1115-1119. https://doi.org/10.1016/j.knee.2014.08.001

20.Grabiner MD, Weiker GG. Anterior cruciate ligament injury and hamstrings coactivation. Clinical Biomechanics, 1993; 8(4): 215-219. https://doi.org/10.1016/0268-0033(93)90017-C

21.Pamukoff DN, Pietrosimone BG, Ryan ED, Lee DR, Blackburn JT. Quadriceps function and hamstrings coactivation after anterior cruciate ligament reconstruction. Journal of Athletic Training, 2017; 52(5): 422-428. https://doi.org/10.4085/1062-6050-52.3.05 
Information about the authors:

Fahri Safa Çinarli; (corresponding author); https://orcid.org/0000-0002-7552-367X; safa.cinarli@gmail.com; Inonu University, Faculty of Sport Sciences, Department of Movement and Training Science, Malatya, Turkey.

Sena Çinarli; https://orcid.org/0000-0003-0671-1762; senaa_siss@hotmail.com; Inonu University, Faculty of Physical Therapy and Rehabilitation, Malatya, Turkey.

Emin Kafkas; https://orcid.org/0000-0002-3962-6428; mkafkas1983@gmail.com; Inonu University, Faculty of Sport Sciences, Department of Movement and Training Science, Malatya, Turkey..

Cite this article as:

Çinarli FS, Çinarli S, Kafkas E. Comparison of thigh muscle activations in single leg exercises: bench squat, step-up, airborne lunge. Pedagogy of Physical Culture and Sports, 2021;25(6):342-348.

https://doi.org/10.15561/26649837.2021.0601

This is an Open Access article distributed under the terms of the Creative Commons Attribution License, which permits unrestricted use, distribution, and reproduction in any medium, provided the original work is properly cited (http://creativecommons.org/licenses/by/4.0/deed.en).

Received: 15.06 .2021

Accepted: 19.08.2021; Published: 30.12.2021 